\title{
Serological Positivity against Selected Flavivirus and Alpha- virus in Free-ranging Bats and Birds from Costa Rica Evidence Exposure to Arboviruses Seldom Reported Locally in Humans
}

Daniel Felipe Barrantes Murillo', Marta Piche-Ovares ${ }^{2-3}$, José Carlos Gamboa-Solano ${ }^{2}$, Luis Mario Romero ${ }^{1}$, Claudio Soto-Garita ${ }^{2}$, Alejandro Alfaro-Alarcón ${ }^{1}$, Eugenia Corrales-Aguilar ${ }^{2 *}$

1 Pathology Department, Universidad Nacional, Costa Rica, 40104, Heredia, Costa Rica; luis.romero.vega@una.cr, alejandro.alfaro.alarcon@una.cr

2 Virology-CIET (Research Center for Tropical Diseases), Faculty of Microbiology, University of Costa Rica, 11501 San José, Costa Rica, jose.gamboasolano@ucr.ac.cr, maria.piche.ovares@una.ac.cr, csoto@inciensa.sa.cr, eugenia.corrales@ucr.ac.cr,

3 PIET (Tropical Disease Research Program), Department of Virology, School of Veterinary Medicine, Universidad Nacional Costa Rica 40104, Heredia, Costa Rica maria.piche.ovares@una.ac.cr

4 Current Address: Department of Pathobiology, College of Veterinary Medicine, Auburn University, 36832, Alabama, USA; dfb0014@auburn.edu

* Correspondence: eugenia.corrales@ucr.ac.cr (E.C.A)

\begin{abstract}
Arboviruses have two ecological transmission cycles, sylvatic and urban. For some, the sylvatic cycle has not been thoroughly described in America. To study the role of wildlife in a putative sylvatic cycle, we sampled free-ranging bats and birds in two arbovirus endemic locations and analyzed them using molecular, serological, and histological methods. No current infection was detected, and no significant arbovirus-associated histological changes were observed. Neutralizing antibodies were detected against selected arboviruses. In bats, positivity in $34.95 \%$ for DENV-1, $16.26 \%$ for DENV-2, 5.69\% for DENV-3, 4.87\% for DENV-4, $2.43 \%$ for WNV, $4.87 \%$ for SLEV, $0,81 \%$ for YFV, $7.31 \%$ for EEEV, and $0.81 \%$ for VEEV was found. Antibodies against ZIKV were not detected. In birds, PRNT results were positive against WNV in $0.80 \%$, SLEV in $5.64 \%$, EEEV in $8.4 \%$, and VEEV in $5.63 \%$. An additional retrospective PRNT analysis was performed using bat samples from three additional DENV endemic sites resulting in a $3.27 \%$ prevalence for $\mathrm{WNV}$ and $1.63 \%$ for SLEV. Interestingly one sample resulted unequivocally WNV positive confirmed by serum titration. These results suggest that free-ranging bats and birds are exposed to not currently reported hyperendemic-human infecting Flavivirus and Alphavirus, however, their role as reservoirs or hosts is still undetermined.
\end{abstract}

Keywords: Arbovirus, bats, birds, wildlife, Costa Rica

\section{Introduction}

Arboviruses are zoonotic infectious agents transmitted by hematophagous vectors [1]. More than 135 different types of arbovirus are responsible for clinical infections in humans [1]. Most of these agents are ARN viruses belonging to different genera such as Flaviviruses and Alphaviruses [1]. Flaviviruses(family Flaviviridae) such as Dengue Virus (DENV), Zika Virus (ZIKV), West Nile Virus (WNV), Saint Louis Encephalitis Virus (SLEV), and Yellow Fever Virus (YFV), have a positive-sense single-stranded RNA genome of approximately $10.8 \mathrm{~kb}$ which encodes for 3 structural proteins (E (envelope), C (Capsid), PrM (pre-membrane)) and 7 non-structural proteins (NS) (NS1, NS2a, NS2b, 
NS3, NS4a, NS4b, and NS5) [2,3]. Alphaviruses, specifically the ones that belong to the family Togaviridae, such as Eastern Equine Encephalitis Virus (EEEV) and Venezuelan Equine Encephalitis Virus (VEEV) have a positive-sense single-stranded ARN genome of approximately $11.8 \mathrm{~kb}$, which encodes 4 non-structural proteins (nsP1-4) and 5 structural proteins (C, E3, E2, 6K, and E1) [4-6].

Arboviruses are maintained in nature through two different transmission cycles, sylvatic and urban [7]. In a sylvatic transmission cycle, a vertebrate reservoir or host capable of maintaining high levels of viremia to become a source of infection for the arthropod vectors is required [7]. In Costa Rica, clinical infections caused by DENV, CHIKV, and ZIKV in human beings have been reported circulating every year in a sustained manner [8-10]. Also, there is serological evidence of SLEV, VEEV, EEEV, and YFV in some wild and domestic animals $[11,12,13]$, and molecular evidence of other arboviruses as well [14]. Thus, Costa Rica is a hyperendemic country for the presence of arbovirus and there is a continuous increasing interest in the search for possible reservoirs in wildlife.

Bats and birds have a wide distribution and diversity in Costa Rica. Bats represent $45 \%$ of the total species of mammals in the country and encompass 115 different species belonging to 9 different families [15]. Costa Rica also possesses an extraordinary diversity of birds, over 922 different species have been reported in the territory, including also migratory birds [16].

For some arboviruses, birds are involved in the sylvatic cycle [17]. Furthermore, bats have been also implicated in maintaining arboviruses in the wild, though inconclusively [18].

To study the role of wildlife in a putative sylvatic cycle in our country, we sampled free-ranging bats and birds in two arbovirus endemic locations and analyzed them using molecular, serological, and histological methods. No current infection was detected, and no significant arbovirus-associated histological changes were observed, though neutralizing antibodies were detected against several hyperendemic arboviruses but also several non-currently circulating in humans arboviruses in our country such as WNV. Our results suggest that free-ranging bats and birds are exposed to not hyperendemic-human infecting Flavivirus and Alphavirus, however, their role as reservoirs or hosts is still undetermined.

\section{Materials and Methods}

Between August 2017 and November 2018, and extensive sampling was performed in two different locations in Costa Rica, Santa Cruz, Guanacaste $\left(10^{\circ} 16^{\prime} 00^{\prime \prime} \mathrm{N} 85^{\circ} 39^{\prime} 00^{\prime \prime} \mathrm{W}\right)$ and Talamanca, Limón $\left(09^{\circ} 44^{\prime} 28^{\prime \prime} \mathrm{N}, 82^{\circ} 50^{\prime} 46^{\prime \prime} \mathrm{W}\right)$ (Figure 1). At each site, eight households (sampling units) were selected and sampled. Birds and bats were collected using mist nets between 15:00-17:00 and 18:00-20:00, respectively. At least five birds and ten bats from each sampling unit were taxonomically identified $[19,20]$ measured, weighed, and euthanized by an intramuscular anesthesia overdose [21] (Supplementary Tables 1-4). All the protocols were approved by the Institutional Committee for the Care and Use of Animals from the University of Costa Rica (CICUA-042-17), Committee of Biodiversity of the University of Costa Rica, and the collection permits from the National System of Conservation Areas (SINAC ACT-PIM-070-17, R-SINAC-PNI-ACLAC-054-2018). 
Figure 1. A. Geographic locations of the sampling sites in Costa Rica, the squares indicate Santa Cruz (Northern Pacific) $\left(10^{\circ} 16^{\prime} 00^{\prime \prime} \mathrm{N} 85^{\circ} 39^{\prime} 00^{\prime \prime} \mathrm{W}\right)$ and Talamanca (Southern Caribbean) $\left(09^{\circ} 44^{\prime} 28^{\prime \prime} \mathrm{N}, 82^{\circ} 50^{\prime} 46^{\prime \prime} \mathrm{W}\right)$. B-C. Each sampling unit is highlighted in these two regions. Maps Source: Map data (02021 Imagery (2021 TerraMetrics and CNES/ Airbus, Lansat/ Copernicus, Maxar Technologies, US Geological Survey from GoogleMaps.
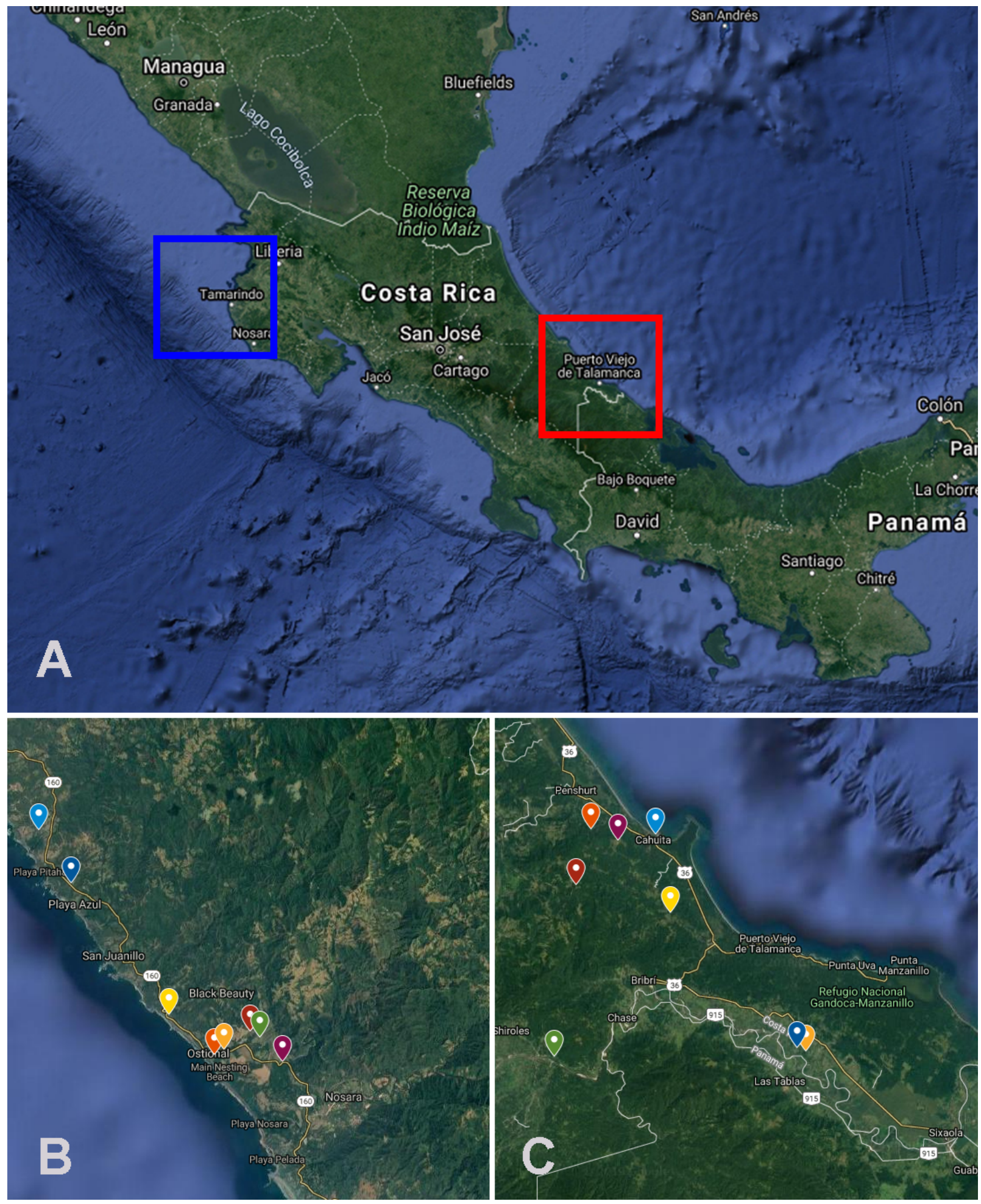

After the euthanasia, a blood sample was taken from the heart. The sample was centrifuged, and the serum was stored at $4^{\circ} \mathrm{C}$ until it arrived at the laboratory. Also, a complete necropsy procedure was performed in each animal; all tissues were collected and placed in $10 \%$ buffered formalin. Additionally, individual samples of lung, spleen, heart, 
kidney, liver, brain, eye, knee, intestine, stomach (ventriculus/proventriculus), urinary bladder, and gonads were taken and placed in RNAlater (Life Technologies, Thermo Fisher Scientific Inc.) and held at $-80^{\circ} \mathrm{C}$. A pool of organs also was taken and placed in RNA later. RNA extraction was performed using TRIzol reagent (Invitrogen, Carlsbad CA, USA), following the manufacturer's instructions. Complementary DNA (DNAc) was obtained using RevertAidTM RT Kit K\#1691 (Thermo Scientific, MA, USA) according to the manufacturer's instructions.

For the identification of viral ARN, a generic RT-PCR using the protocol designed for amplification of partial NS5 segment of Flavivirus [22] and the partial amplification of nsP4 for Alphavirus [23] was performed. The amplicons were analyzed by electrophoresis and interpreted using the equipment QIAxcel Advanced (QIAGEN) and the Software QIAxcel ScreenGel 1.6.0.

A plaque reduction neutralization test (PRNT) assay was performed with a unique serum dilution (1:10) of each sample to detect neutralizing antibodies. Neutralization assays were performed in 96-well, flat-bottomed tissue culture plates because of the limited sera volume. Briefly, each sample was heat-inactivated for 30 minutes at $56^{\circ} \mathrm{C}$.). The serum was diluted 1:5 in MEM with 2\% FBS. Each virus were prepared to an estimated end of 10 UFP/well (WNV (YFV 17D/WNV Flamingo 383-99), DENV 1-4 (YFV 17D/DENV-1 PUO 359, YFV 17D/DENV-2 218, YFV 17D/DENV-3 PaH881/88, YFV 17D/DENV-4 1228), ZIKV (ATCC® VR-748), SLEV (YFV 17D/SLEV CorAn 9124) EEEV-Sindbis, VEEV (TC83 252296), Zika VR 1848 ATCC and YFV 17D). The virus-serum mix was incubated $1 \mathrm{~h}$ at $37^{\circ} \mathrm{C}$. Then, $50 \mu \mathrm{L}$ of this mixture was added to Vero Cells monolayer previously seeded and incubated for $1 \mathrm{~h}$ at $37^{\circ} \mathrm{C}$ in a $5 \% \mathrm{CO}_{2}$ atmosphere. After adsorption, the mixture was removed from Vero cells, and $100 \mu \mathrm{L}$ of MEM with $2 \%$ of FBS and $1.5 \%$ of carboxymethylcellulose was added. (Sigma-Aldrich, USA). Plates were incubated for $72 \mathrm{~h}$ at $37^{\circ} \mathrm{C}$ in a $5 \%$ $\mathrm{CO}_{2}$ atmosphere. After the incubation period, MEM containing CMC was removed, and plates were fixed during an hour with formalin (3.7\%) and stained with crystal violet (1\%). A $90 \%$ reduction of foci number relative to the average of the viral control (no sera) was considered positive.

A second retrospective PRNT assay was performed using serum samples from 61 bats previously collected in three different endemic areas: Sarapiquí, Heredia (10 24'20" N, 84 8'3" W) Nicoya, Guanacaste (10 9'42" N, $85^{\prime 2} 26^{\prime} 48^{\prime \prime}$ W) and Valle Central (9 5'42" N, 84 8'35" W) between 2013-2014 [24]. PRNT assays were performed using 1:20 dilution, using the same methodology and viruses (except ZIKV, since it was not yet introduced to the country) described above. These samples from the retrospective study were then categorized as moderately positive (cutoff of $\geq 50 \%$ PRNT on Vero cells) and highly positive (cutoff of $\geq 90 \%$ PRNT on Vero cells). Highly positive samples ( $>90 \%$ ) against WNV were submitted for serum titration using serial dilutions from 1:40 to 1:640.

Furthermore, tissue samples fixed in 10\% neutral buffered formalin were embedded in paraffin, sectioned at $3 \mu \mathrm{m}$, and routinely stained with Hematoxylin and Eosin. Complete histopathological analysis of all tissues was done, characterizing the inflammatory infiltrate, severity, chronicity, and distribution of the lesions. The histopathological analysis aimed to find inflammatory lesions within the central nervous system, joints, reproductive tract, liver, spleen, previously reported in experimental animal models and the natural viral infection [25-29].

\section{Results}

For this study, a total of 144 bats (75 males and 69 females) representing 5 families and 26 species were collected (Supplementary Tables 1-2). Due to the limited amount of serum, PRNT 1:10 dilution was performed only in 123 serum samples from bats. Neutralizing antibodies were detected against Dengue (DENV 1-4), Zika Virus (ZIKV), Yellow Fever Virus (YFV), West Nile Virus (WNV), Saint Louis Encephalitis Virus (SLEV), Eastern Equine Encephalitis Virus (EEEV), and Venezuelan Encephalitis Virus (VEEV). In bats, 34.95\% (43/123) for DENV-1, 16.26\% (20/123) for DENV-2, 5.69\% (7/123) for DENV- 
3, 4.87\% (6/123) for DENV-4, 2.43\% (3/123) for WNV, 4.87\% (6/123) for SLEV, $0.81 \%(1 / 123)$ for YFV, $7.31 \%(9 / 123)$ for EEEV and $0.81 \%(1 / 123)$ for VEEV were found as positive. Antibodies to ZIKV were not detected. Cross-reactions among the different types of Flaviviruses and Alphaviruses were present in $39.28 \%$ of the positive samples $(22 / 56)$ (Table 1). A percentage $(17.88 \%)$ of the bats were exclusively positive for DENV-1 (22/123), 3.25\% were exclusively positive for DENV-2 (4/123), 0.81\% for DENV-3 (1/123), 0.81\% for DENV4 (1/123), $0.81 \%$ for WNV (1/123), 3.25\% for EEEV (4/123) and 0.81\% for VEEV (1/123) (Table 2). No individuals with neutralizing antibodies exclusively against SLEV and YFV were found.

Table 1. Species of bats presenting serological cross-reaction among viruses (Flaviviruses and Alphaviruses) analyzed in the PRNT 1:10 dilution.

\begin{tabular}{lll}
\hline Species & Sex & $\begin{array}{l}\text { Positive results in PRNT 1:10 (putative } \\
\text { cross reactions) }\end{array}$ \\
\hline Glossophaga soricina & Female & DENV-1/ DENV-3 \\
Artibeus jamaicensis & Female & DENV-1 DENV-2 \\
Artibeus phaeotis & Female & DENV-1 DENV-2 DENV-4 \\
Artibeus phaeotis & Female & DENV-1 DENV-2 DENV-4 \\
Phyllostomus discolor & Male & DENV-1 EEEV \\
Phyllostomus discolor & Female & DENV-1 DENV-2 DENV-3 EEEV \\
Noctilio albiventris & Male & DENV-1 EEEV \\
Artibeus lituratus & Female & DENV-1/ DENV-2/ DENV-3 \\
Artibeus jamaicensis & Male & DENV-1 DENV-2 EEEV \\
Artibeus phaeotis & Male & DENV-1 DENV-2 WNV SLEV \\
Carollia perspicillata & Male & DENV-1 DENV-2 \\
Artibeus watsoni & Male & DENV-1 DENV-2 DENV-3 DENV-4 \\
Artibeus jamaicensis & Female & DENV-1 SLEV \\
Carollia perspicillata & Male & DENV-1 DENV-2 \\
Glossophaga soricina & Female & DENV-1 DENV-2 DENV-3 DENV-4 \\
Artibeus jamaicensis & Female & DENV-1 DENV-2 YFV WNV \\
Artibeus jamaicensis & Female & SLEV, EEEV \\
Uroderma convexum & Female & DENV-1 DENV-2 DENV-3 SLEV \\
Uroderma convexum & Female & DENV-1 DENV-2 SLEV \\
Carollia perspicillata & Female & DENV-1 DENV-2 \\
Artibeus jamaicensis & Male & DENV-1 DENV-2 \\
Artibeus jamaicensis & Male & DENV-1 DENV-4 SLEV \\
\hline
\end{tabular}


Table 2. Species of bats with neutralizing antibodies exclusive (specific) against Flaviviruses or Alphaviruses at PRNT 1:10 dilution.

\begin{tabular}{|c|c|c|}
\hline Species & Sex & Exclusive reaction in PRNT 1:10 \\
\hline Uroderma convexum & Male & DENV-1 \\
\hline Artibeus phaeotis & Male & DENV-1 \\
\hline Desmodus rotundus & Female & DENV-1 \\
\hline Sturnira parvidens & Female & DENV-1 \\
\hline Artibeus jamaicensis & Male & DENV-1 \\
\hline Chiroderma salvini & Male & DENV-1 \\
\hline Ptenorotus mesoamericanus & Female & DENV-1 \\
\hline Carollia perspicillata & Female & DENV-1 \\
\hline Uroderma convexum & Male & DENV-1 \\
\hline Glossophaga soricina & Male & DENV-1 \\
\hline Loncophylla robusta & Female & DENV-1 \\
\hline Myotis nigricans & Female & DENV-1 \\
\hline Phyllostomus discolor & Male & DENV-1 \\
\hline Artibeus jamaicensis & Male & DENV-1 \\
\hline Glossophaga soricina & Male & DENV-1 \\
\hline Carollia perspicillata & Female & DENV-1 \\
\hline Carollia perspicillata & Male & DENV-1 \\
\hline Artibeus jamaicensis & Female & DENV-1 \\
\hline Uroderma convexum & Male & DENV-1 \\
\hline Carollia castanea & Female & DENV-1 \\
\hline Rhogeessa io & Female & DENV-1 \\
\hline Uroderma convexum & Male & DENV-1 \\
\hline Glossophaga soricina & Female & DENV-2 \\
\hline Artibeus lituratus & Male & DENV-2 \\
\hline Artibeus watsoni & Male & DENV-2 \\
\hline Glossophaga soricina & Male & DENV-2 \\
\hline Artibeus phaeotis & Female & DENV-3 \\
\hline Desmodus rotundus & Female & DENV-4 \\
\hline Carollia castanea & Female & WNV \\
\hline Carollia perspicillata & Female & EEEV \\
\hline Phyllostomus discolor & Male & EEEV \\
\hline Phyllostomus discolor & Male & EEEV \\
\hline Artibeus jamaicensis & Male & EEEV \\
\hline Carollia perspicillata & Male & VEEV \\
\hline
\end{tabular}

We expanded the PRNT assays in 61 of 241 bat samples that have previously been tested solely against DENV in one of our previous studies, in which we reported a $21.2 \%$ DENV seropositivity in bats [24]. Now these sera were retrospectively assessed once again but against other Flaviviruses. The preliminary 1:20 dilution results showed that $11.4 \%$ (7/61) have neutralizing antibodies against WNV and 14.75\% (9/61) against SLEV (Table 3 ). The samples were then categorized as moderately positive (cutoff of $\geq 50 \%$ PRNT ) and highly positive (cutoff of $\geq 90 \%$ PRNT). From all the samples analyzed, $19.67 \%$ (12/61) were classified as moderately positive and only $4.91 \%$ (3/61) were highly positive against SLEV and WNV. Of the moderately positive samples, 58\% (7/12) present serological cross-reaction against at least 1 serotype of DENV (Table 3 ). Highly positive samples were tested by further dilution of sera when it was available. Surprisingly and interestingly, a highly positive sample against WNV presented a neutralizing titer of $>1: 640$ for WNV with a titer of $<1: 40$ for SLEV and DENV 1-4. After this reassessment and due to the observed serological cross-reaction between arboviruses, only highly positive samples were then considered truly seropositive for WNV or SLEV. Therefore, overall prevalence exclusively for 
WNV and SLEV in these bats was 3.27\% (2/61) and 1.63\% (1/61) respectively. No WNV or SLEV circulation in these areas (Valle central) has been so far reported in humans or wildlife.

Table 3. Species of bats categorized as moderately or highly neutralizing samples against SLEV and WNV by PRNT showed cross-reaction with other Flaviviruses.

\begin{tabular}{|c|c|c|}
\hline Species & Sex & Cross reactions in PRNT 1:20 \\
\hline Molossus sinaloe & Female & SLEV, DENV-3** \\
\hline Molossus sinaloe & Female & WNV, DENV-3** \\
\hline Molossus sinaloe & Female & SLEV, DENV-3** \\
\hline Molossus sinaloe & Female & WNV, DENV-3** \\
\hline Molossus sinaloe & Female & $\mathbf{W N V}^{*}, \mathrm{DENV}-3^{* *}$ \\
\hline Molossus sinaloe & Male & WNV, SLEV, DENV-2**, DENV-3** \\
\hline Molossus sinaloe & Male & SLEV, DENV-3** \\
\hline Molossus sinaloe & Male & SLEV $, \mathrm{DENV}-1^{* *}, \mathrm{DENV}-2^{* *}, \mathrm{DENV} 4^{* *}$ \\
\hline Uroderma bilobatum & Male & WNV, DENV-3** \\
\hline
\end{tabular}

A total of 140 birds were collected (89 males and 51 females), representing 37 genera and 43 species (Supplementary Tables 3-4). Serum neutralization analysis (PRNT 1:10) was performed against WNV and SLEV in 124 serum samples and against EEEV and VEEV in 71 serum samples due to sample volume constraints. In birds, PRNT resulted positive against WNV in $0.80 \%(1 / 124)$, SLEV in 5.64\% (7/124), EEEV in 8.4\% (6/71), and VEEV in 5.63\% (4/71). Cross-reactions were also observed in 2 individuals presenting antibodies against EEEV and VEEV (Table 4).

Table 4. Species of birds seropositive in the PRNT 1:10 dilution against selected arbovirus.

\begin{tabular}{lll}
\hline Species & Sex & PRNT 1:10 Result \\
\hline Aimophila ruficauda & Male & EEEV \\
Turdus grayi & Male & SLEV \\
Pitangus sulphuratus & Female & EEEV \\
Quiscalus mexicanus & Female & VEEV \\
Icterus pustulatus & Male & EEEV/VEEV \\
Icterus pustulatus & Male & EEEV \\
Campylorhynchus rufinucha & Female & WNV \\
Pitangus sulphuratus & Male & VEEV \\
Crotophaga sulcirostris & Male & EEEV \\
Myozetetes similis & Male & SLEV \\
Empidonax virescens* & Male & SLEV/EEEV/VEEV \\
\hline
\end{tabular}

*Migratory species.

All RT-PCR analyses performed in both blood and pool tissues collected from bats and birds were negative for the detection of viral ARN from flaviviruses and alphaviruses. Histopathological findings were nonspecific, inflammatory, and degenerative changes frequently associated with the presence of diverse parasites (protozoa, metazoans). Thus, histological analysis of tissues did not show any significant findings related to arboviral infections 


\section{Discussion and Conclusions}

The sylvatic cycle from some arboviruses has not been thoroughly described. Birds have been implicated in participating in the virus cycle of some arboviruses [30-33]. Nevertheless, even though representing one of the most abundant mammals and diverse species in many tropical and neotropical areas, bats are still not directly implicated as part of the virus cycle of the most important arboviruses infecting humans such as DENV, ZIKV, and WNV, but have been described as accidental hosts $[18,24]$. In many areas, the identification of wildlife susceptible to infection and thus been able to participate as hosts or reservoirs is not fully elucidated, and surely, just identifying exposure does not translate into being part of the virus cycle. Nevertheless, because of the importance of this identification and to further study the role of wildlife in a putative sylvatic cycle in our country, we sampled free-ranging bats and birds in two arbovirus endemic locations and analyzed them using molecular, serological, and histological methods. No current infection was detected, and no significant arbovirus-associated histological changes were observed. Though neutralizing antibodies were detected against several arboviruses. These results demonstrate that free-ranging bats and birds in our country are exposed to not hyperendemic-human infecting Flaviviruses and Alphaviruses.

PRNT is the gold standard for the serological diagnosis of previous arboviral infection [17]. Using PRNT with chimeric viruses we demonstrated the presence of antibodies against several Flaviviruses (DENV1-4, SLEV, YFV, WNV) and Alphaviruses (EEEV, VEEV) in bats and birds in our country. However, as expected cross-reactions with different Flaviviruses and Alphaviruses were prevalent in our microneutralization assays. Cross-reactions make it difficult to establish unequivocally the exposure to the etiologic virus. This phenomenon is explained by the similarities between the immunodominant epitopes within the family Flaviviridae and Togaviridae [17]. The envelope structural glycoprotein is the main target for the neutralizing antibodies in Flaviviridae and E2 structural protein for Togaviridae [34]. To determine against which specific arbovirus the animal was exposed, titration of the serum is necessary [17]. Unfortunately, the scant amount of serum available in most of our samples did not allow this. However, studies suggest that the serological evidence in a PRNT allows determining that bats were exposed against a given arbovirus in a period between 10 days to months before the analyses [17]. In the retrospective serological study with samples that were preliminary positive for DENV, serum titration allowed the identification and confirmation of a single bat from an urban area (Valle Central) unequivocally positive to WNV (Molossus sinaloe) with a titer $>1: 640$ [35].

On the other hand, viral RNA was not evidenced in the blood or tissues from any of the sampled animals. Because arboviral infections are generally transient, the chance of detecting viral RNA from sera or tissues is extremely low [36]. In this study, we did not detect any active viremia in bats and birds.

This is not the first evidence of arboviruses in bats in Costa Rica. A previous study demonstrated serological prevalence in $21.2 \%$ (51/241) and positivity by RT-PCR in $8.8 \%$ (28/318) bats for DENV RNA [24]. The retrospective PRNT assays were performed with the samples obtained from this previous project. However, these results contrast with our findings, in which we cannot evidence viral RNA in the animal blood and/or tissues. In the previous report, it was speculated ingestion of mosquitoes as the route of infection, since most of the collected samples were from insectivorous animals. Bats as a dead-end host for DENV were then suggested [24]. The majority of the bats collected in this study are frugivorous, and we did not detect any viral RNA in any organ including intestines. Furthermore, during histological evaluation on the intestines no evidence of ingested arthropods was observed. Thus, infection through the ingestion of mosquitoes might be unlikely. Mosquito feeding therefore seems to be the more plausible route of infection. 
In other locations in the American continent, bats have been subjected to extensive evaluations for arbovirus infection with different outcomes. In Brazil, 103 samples from free-ranged bats were analyzed via nested-PCR and hemagglutination inhibition test. All the samples were negative for arbovirus; thus, the authors concluded that bats do not constitute a reservoir for these viruses in the studied areas [37]. In Trinidad, a serological analysis demonstrated a prevalence of $2.9 \%$ (11/384) against VEEV using an ELISA in bats [36]. In Colombia, VEEV viral RNA and antigen detection were demonstrated via RT-PCR and immunohistochemistry (IHC)in the brain tissues from two frugivorous bats, suggesting that these species might be hosts for this zoonotic agent [38]. Finally, a new strain of encephalitogenic VEEV (Tonate Virus) was isolated from bats in French Guiana [39]. Neutralizing antibodies against SLEV have been reported in bats from Ohio, USA? [40]. WNV has never been reported in bats in Central America [41], there are only two reports of the presence of neutralizing antibodies against WNV in the USA $[42,43]$. YFV and ZIKV infection has not been reported so far in bats in America [44]. We found only one bat with neutralizing antibodies against YFV, but with a clear cross-reaction against DENV-1, DENV-2 and WNV. Only one of the three bats positive against WNV was positive exclusively and unequivocally to this agent. In the retrospective assay, one Molossus sinaloe was confirmed through serum titration (1:640) as seropositive against WNV. Thus, this is the first evidence of WNV exposure in bats in Central America.

This is the first time that SLEV, WNV, EEEV, and VEEV exposure have been demonstrated in wild birds in Costa Rica. Overall reported seroprevalence against those agents is low in America. A study conducted in Yucatán México revealed a seroprevalence of $4.3 \%(11 / 257)$ against WNV through ELISA [30]. In Trinidad, one study reported a seroprevalence of $1.4 \%$ (2/140) against SLEV through hemagglutinin inhibition test [45]. WNV and SLEV can cross-react [31] and serum titration was not performed. Only one species, Empidonax virescens is migratory and have neutralizing antibodies against SLEV and cross reaction between VEEV and EEEV: suggesting a non-local exposure. None of the species identified in this study has been reported as a reservoir for SLEV and WNV [17]. Therefore, we conclude that some of our sampled birds were exposed to these viruses, but since we did not detect any active viremia, we were not able to identify the putative reservoirs for these viruses in our country. Thus, further sampling efforts in exemplar numbers and areas must be conducted.

Taken together, we can conclude that free-ranging bats and birds are exposed to not currently reported hyperendemic-human infecting Flaviviruses such as WNV and some Alphaviruses, however, their role as reservoirs or hosts is still undetermined. They may have a role as a dead-end host, but no evidence supports that the sampled species during this study might function as reservoirs.

Author Contributions: Conceptualization, E.C.A.; methodology, E.C.A., D.F.B.M., M.P.O., J.C.G.S., L.M.R., C.S.G., A.A.A.; software, D.F.B.M.; validation, E.C.A., D.F.B.M., M.P.O.; formal analysis, E.C.A., D.F.B.M., investigation, E.C.A., D.F.B.M.; resources, E.C.A; data curation, D.F.B.M., J.C.G.S., M.P.O; writing-original draft preparation, D.F.B.M., E.C.A; writing-review and editing, D.F.B.M., E.C.A., M.P.O., J.C.G.S., L.M.R., C.S.G., A.A.A., visualization, E.C.A; supervision, E.C.A.; project administration, E.C.A; funding acquisition, E.C.A. All authors have read and agreed to the published version of the manuscript.

Funding: This study was financially supported by EU Horizon 20/20 ZIKALLIANCE (grant agreement 734548), National Council of Rector (Special Fund of Higher Education, FEES-B7-362), and the Postgraduate Studies System from the University of Costa Rica (UCR).

Institutional Review Board Statement: The study and associated protocols were designed based on national ethical legislation and approved by the Institutional Committee of Care and Use of Animals of the University of Costa Rica (CICUA-042-17), Committee of Biodiversity of the University 
of Costa Rica (VI-2994-2017), National System of Conservation Areas (SINAC): Tempisque Conservation Area (Oficio-ACT-PIM-070-17), La Amistad-Caribe Conservation Area (M-PC-SINAC-PNIACLAC-047-2018). The survey did not involve endangered or protected species.

Acknowledgments: We thank Francisco Vega, Geovanny Vargas, Carlos Vargas, Laura Orozco, Luis Bermúdez, and Laura Alvarado who supplied technical assistance for the processing of the samples. We thank Dr. Juan Alberto Morales, Dr. Julia Lankton, Dr. Saskia Keller, and Dr. Valerie Schaern-Boschler for the assistance during the histological analysis of the tissues. We thank the Arbovirus Reference Collection (ARC) from the Centers for Disease Control and Prevention, National Center for Emerging and Zoonotic Infectious Diseases, Division of Vector-Borne Diseases, Arboviral Diseases Branch, Diagnostic and Reference Team, Reference and Reagent Laboratory and Sanofi Pasteur for the chimeric viruses contribution and the Gorgas Institute from Panama for the alphaviruses contribution.

Conflicts of Interest: The authors declare no conflict of interest.

\section{References}

1. Mayer, S.V.; Tesh, R.B.; Vasilakis, N. The emergence of arthropod-borne viral diseases: a global prospective on Dengue, Chikungunya and Zika Fevers. Acta Trop. 2016, 166, 155-163.

2. Chen, C.I.; Clark, D.C.; Pesavento, P.; Lerche, N.W.; Luciw, P.A.; Reisen, W.K.; Brault, A.C. Comparative Pathogenesis of Epidemic and Enzootic Chikungunya Viruses in a Pregnant Rhesus Macaque Model. Am. J. Trop. Med. Hyg. 2010, 83 (6), 1249_ 1258.

3. Vasilakis, N.; Weaver, S.C. Flavivirus transmission focusing on Zika. Curr Opin Virol. 2017, 22, 30-35.

4. Singh, S.K.; Unni, S.K. Chikungunya virus: host pathogen interaction. Rev Med Virol. 2011, 21, 78-88.

5. Tsetsarkin, K.A.; Chen, R.; Weaver, S.C. Interspecies transmission and chikungunya virus emergence. Curr Opin Virol. 2016, $16,143-150$.

6. Krutikov, M.; Manson, J. Chikungunya Virus Infection: An Update on Joint Manifestations and Management. Rambam. Maimonides. Med. J. 2016, 7 (4), e0033.

7. Kuno, G.; Mackenzie, J.S.; Junglen, S.; Hubálek, Z.; Plyusnin, A.; Gubler, D.J. Vertebrate Reservoirs of Arboviruses: Myth, Synonym of Amplifier, or Reality? Viruses. 2017, 13;9 (7), e185.

8. Ministerio de Salud. Análisis de la situación de salud: Dengue 2017. https://www.ministeriodesalud.go.cr/index.php/vigilancia-de-la-salud/analisis-de-situacion-de-salud/3280-dengue-2017/file (accessed on 15 September 2017).

9. Ministerio de Salud. Análisis de la situación de salud: Zika 2017. https://www.ministeriodesalud.go.cr/index.php/vigilanciade-la-salud/analisis-de-situacion-de-salud/3278-zika-2017/file (accessed on 15 September 2017).

10. Ministerio de Salud. Análisis de la situación de salud: Chikungunya 2017. https://www.ministeriodesalud.go.cr/index.php/vigilancia-de-la-salud/analisis-de-situacion-de-salud/3279-chikungunya-2017/file (accessed on 15 September 2017).

11. Medlin, S.; Deardorff, E.R.; Hanley, C.S.; Vergneau-Grosset, C.; Siudak-Campfield, A.; Dallwig, R.; Travassos da Rosa, A.; Tesh, R.B.; Martin, M.P.; Weaver, S.C.; Vaughan, C.; Ramirez, O.; Sladky, K.K.; Paul-Murphy, J. Serosurvey of Selected Aboviral Pathogens in Free-Ranging, Two-Toed Sloths (Choloepus hoffmani) and Three-Toed Sloths (Bradypus variegatus) in Costa Rica, 2005-07. J Wildl Dis. 2016, 52(4), 883-892.

12. Berger, S. Yellow Fever: Global Status, 2019 ed.; Gideon E-Book Series: Gideon Informatic Inc. Los Angeles, CA, USA, 2019; pp. 70-71.

13. Jiménez, C; Romero, M; Piche, M; Baldi, M; Alfaro, A; Chaves, A; Morales, J; león, B; Hutter, S; Corrales-Aguilar, E. Arboviral encephalitis in Costa Rican horses: 2009-2016. Int. J. Infect. Dis. 2016, 53, 153.

14. León, B; Jiménez, C; González, R; Ramirez-Carvajal, L. First Complete Coding Sequence of a Venezuelan Equine Encephalitis Virus Strain Isolated from an Equine Encephalitis Case in Costa Rica. Microbiol. Resour. Announc. 2019, 5;8(36):e00672-19.

15. Rodríguez-Herrera, B.; Ramírez-Fernández, J.D.; Villalobos-Chaves, D.; Sánchez, R. Actualización de la Lista de Especies de Mamíferos Vivientes de Costa Rica. Mastozool. Neotrop. 2014, 21(2), 275-289.

16. Garrigues, Richard., P. CaMale-Varela, M. Montoya, P. O’Donnell, O. Ramírez-Alán, J. Zook. (2018, January 4). Lista Oficial de las Aves de Costa Rica - Actualización 2018. Comité de Especies Raras y Registros Ornitológicos de Costa Rica (Comité Científico), Asociación Ornitológica de Costa Rica [PDF File]. Retrieved from https://listaoficialavesdecostarica.wordpress.com/lista-oficial/lista-oficial-online/ 
17. Contigiani, M.A.; Diaz, L.A.; Spinsanti, L.I. General Aspects on Arboviruses. In Arthropod Borne Diseases, 1st ed; BrisolaMarcondes, C., Ed.; Springer International Publishing: Switzerland, 2017; pp. 61-71.

18. Moreira-Soto, A.; Corrales-Aguilar, E. Bats and Flaviviruses. In Bats and Viruses: Current Research and Future Trends, 1st ed; Corrales-Aguilar, E.; Schwemmle, M., Eds; Caister Academic Press: Poole, United Kingdom, 2020; pp. 7-26.

19. Garrigues R, Dean R. The Birds of Costa Rica: A Field Guide. 1 st ed.; Cornell University Press: New York, USA, $2007 ;$ pp. 1-440.

20. York, H.A; Rodríguez-Herrera, B; Laval, R.K.; Timm, R.M.; Lindsay, K.E. Field key to the bats of Costa Rica and Nicaragua, J. Mammal. 2019, 100 (6), 1726-1749.

21. American Veterinary Medical Association. Guidelines for the Euthanasia of Animals: 2013 Edition. https://www.avma.org/sites/default/files/resources/euthanasia.pdf (accessed on 12 November 2020).

22. Scaramozzino, N.; Crance, J.M.; Jouan, A.; Debriel, D.A.; Stoll, F.; Garin, D. Comparison of Flavivirus Universal Primer Pairs and Development of a Rapid, Highly Sensitive Heminested Reverse Transcription-PCR Assay for Detection of Flaviviruses Targeted to a Conserved Region of the NS5 Gene Sequences. J Clin Microbiol. 2001, 39 (5), 1922-1927.

23. Grywna, K.; Kupfer, B.; Panning, M.; Drexler, J.F.; Emmerich, P.; Drosten, C.; Kümmerer, B.M. Detection of All Species of the Genus Alphavirus by Reverse Transcription-PCR with Diagnostic Sensitivity. J. Clin. Microbiol. 2010, 48 (9), $3386-3387$.

24. Vicente-Santos, A.; Moreira-Soto, A.; Soto-Garita, C.; Chaverri, L.G.; Chaves, A.; Drexler, J.F.; Morales, J.A.; Alfaro-Alarcón, A.; Rodríguez-Herrera, B.; Corrales-Aguilar, E. Neotropical bats that co-habit with humans' function as dead-end hosts for dengue virus. PLoS Negl Trop Dis. 2017, 11(5), e0005537.

25. Goupil, B.A.; McNulty, M.A.; Martin, M.J.; McCracken, M.K.; Christofferson, R.C.; Mores, N.C. Novel Lesions of Bones and Joints Associated with Chikungunya Virus Infection in Two Mouse Models of Disease: New Insights into Disease Pathogenesis. PLOS ONE. 2016, 11(5), e0155243.

26. Yu, J.; Liu, X.; Ke, C.; Wu, Q.; Lu, W.; Qin, Z.; He, X.; Liu, Y.; Deng, J.; Xu, S.; Li, Y.; Zhu, L.; Wan, C.; Zhang, Q.; Xia o, W.; Xie, Q.; Zhang, B.; Zhao, W. Effective Suckling C57BL/6, Kunming, and BALB/c Mouse Models with Remarkable Neurological Manifestation for Zika Virus Infection. Viruses. 2017, 9 (7), 165.

27. Sarathy, V.V.; Milligan, G.N.; Bourne, N.; Barrett, A.D. Mouse models of dengue virus infection for vaccine testing. Vaccine. 2015, 33 (50), 7051-7060.

28. Hubálek, Z.; Rudolf, I.; Nowotny, N. Arboviruses pathogenic for domestic and wild animals. Adv Virus Res. $2014 ; 89,201-75$.

29. Senne, D.A.; Pedersen, J.C.; Hutto, D.L.; Taylor, W.D.; Schmitt, B.J.; Panigrahy, B. Pathogenicity of West Nile virus in chickens. Avian Dis. 2000, 44(3), 642-649.

30. Farfán-Ale, J.A.; Blitvich, B.J.; Marlenee, N.L.; Loroño-Pino, M.A.; Puerto-Manzano, F.; García-Rejón, J.E.; Rosado-Paredes, E.P.; Flores-Flores, L.F.; Ortega-Salazar, A.; Chávez-Medina, J.; Cremieux-Grimaldi, J.C.; Correa-Morales, F.; HernándezGaona, G.; Méndez-Galván, J.F.; Beaty, B.J. Antibodies to West Nile virus in asymptomatic mammals, birds, and reptiles in the Yucatan Peninsula of Mexico. Am. J. Trop. Med. Hyg. 2006, 74(5), 908-914.

31. Diaz, A.; Coffey, L.L.; Burkett-Cadena, N.; Day, J.F. Reemergence of St. Louis Encephalitis Virus in the Americas. Emerg. Infect. Dis. 2018, 24(12), 2150-2157.

32. Tan, Y.; Tommy Lam, T.T.Y.; Heberlein Larson, L.A.; Smole, S.C.; Auguste, A.J.; Hennigan, S.; Halpin, R.A.; Fedorova, N.; Puri, V.; Stockwell, T.B.; Shilts, M.H.; Andreadis, T.; Armstrong, P.M.; Tesh, R.B.; Weaver, S.C.; Unnasch, T.R.; Ciota, A.T.; Kramer, L.D.; Das, S.R. 2018. Large-scale complete-genome sequencing and phylodynamic analysis of eastern equine encephalitis virus reveals source-sink transmission dynamics in the UnitedStates. J. Virol. 2018, 92 (12), e00074-18.

33. Kelen V.P.; Downs, J.A.; Unnasch, T.; Stark, L. 2014. A risk index model for predicting eastern equine encephalitis virus transmission to horses in Florida. Appl Geogr. 2014, 1 (48), 79-86.

34. Kim, A.S.; Austin, K.; Gardner, C.L.; Zuiani, A.; Reed, D.S.; Trobaugh, D.W.; Sun, C.; Basore, K.; Williamson, L.E.; Crowe Jr., J.E.; Slifka, M.K.; Fremont, D.H.; Klimstra, W.B.; Diamond, M.S. 2019. Protective antibodies against Eastern equine encephalitis virus bind to epitopes in domains A and B of the E2 glycoprotein. Nat Microbiol. 2019, 4(1): 187-197.

35. World Health Organization. Laboratory Diagnosis for the Infection for Yellow Fever Virus. https://www.paho.org/hq/index.php?option=com docman\&task=doc view\&gid=43571\&Itemid=270\&lang=en (accessed on 15 April 2018).

36. Thompson, N.N.; Auguste, A.J.; Travassos da Rosa, A.P.; Carrington, C.V.; Blitvich, B.J.; Chadee, D.D.; Tesh, R.B.; Weaver, S.C.; Adesiyun, A.A. Seroepidemiology of selected alphaviruses and flaviviruses in bats in Trinidad. Zoonoses Public Health. 2014, 62(1), 53-60.

37. Bittar, C.; Machado, R.R.G.; Comelis, M.T.; Bueno L.M.; Morielle-Versute, E.; Beguelini M.R.; de Souza, R.P.; Nogueira, M.L.; Rahal, P. Lack of serological and molecular evidence of arbovirus infections in bats from Brazil. PLoS One. 2018, 7;13(11):e0207010.

38. Guzmán, C.; Calderón, A.; Oviedo, T.; Mattar, S.; Catañeda, J.; Rodríguez, V.; Moraes-Figueiredo, L.T. Mollecular an celular evidence if natural Venezuelan equine encephalitis virus infection in frugivorous bats in Colombia. Vet World. 2020,13 (3), 495-501.

39. Fischer, C.; Pontier,D.; Filippi-Codaccioni, O.; Pons, J.; Postigo-Hidalgo, I.; Duhayer, J.; Brünink,s.; Drexler, J.F. Venezuelan Equine Encephalitis Complex Alphavirus in Bats, French Guiana. Emerg. Infect. Dis. 2021, 27(4), 1141-1145. 
40. Herbold, J.; Heuschele, W.; Berry, R.; Parsons, M. Reservoir of St. Louis encephalitis virus in Ohio bats. Am. J Vet. Res. 1983 , 44, 1889-1893.

41. Stone, D.; Lyons, A.; Huang, Y.J.; Vanlandingham, D.; Higgs, S.; Blitvich, B.; Adesiyun, A.; Santana, S.; Leiser-Miller, L.; Cheetham, S. Serological evidence of widespread exposure of Grenada fruit bats to chikungunya virus. Zoonoses Public Health. 2018, 65, 505-511.

42. Pilipski, J.D.; Pilipski, L.M.; Risley, L.S. West Nile virus antibodies in bats from New Jersey and New York. J. Wildl. Dis. 2004, 40, 335-337.

43. Davis, A.; Bunning, M.; Gordy, P.; Panella, N.; Blitvich, B.; Bowen, R. Experimental and natural infection of North American bats with West Nile virus. Am. J. Trop. Med. Hyg. 2005, 73(2), 467-469.

44. Fagre, A.C.; Kading, R.C. Can Bats Serve as Reservoirs for Arboviruses? Viruses. 2019, 11(3), e215. 3;11(3).

45. Thompson, N.N.; Auguste, A.J.; Coombs, D.; Blitvich, B.J.; Carrington, C.V.; da Rosa, A.P.; Wang, E.; Chadee, D.D.; Drebot, M.A.; Tesh, R.B.; Weaver, S.C.; Adesiyun, A.A. Serological evidence of flaviviruses and alphaviruses in livestock and wildlife in Trinidad. Vector Borne Zoonotic Dis. 2012, 12(11), 969-978. 